\title{
Seroprevalence of low avidity anti-Toxoplasma IgG in pregnant women and its relationship with their age and contact with cats
}

\author{
Khan, K. ${ }^{1}$, Khan, W..$^{*}$, Khan, T. ${ }^{2}$, Naaz, G. ${ }^{3}$, Naheda, A. ${ }^{1}$ and Aqeel, S. ${ }^{1}$ \\ ${ }^{1}$ Section of Parasitology, Department of Zoology, Aligarh Muslim University, Aligarh, India \\ ${ }^{2}$ Department of Obstetrics and Gynaecology, Jawaharlal Nehru Medical College, Aligarh Muslim University, \\ Aligarh, India \\ ${ }^{3}$ Department of Botany, Aligarh Muslim University, Aligarh, India \\ *Corresponding author e-mail: wajihullahkhan@yahoo.co.in \\ Received 17 February 2020; received in revised form 7 July 2020; accepted 8 July 2020
}

\begin{abstract}
Toxoplasma gondii is a protozoan parasite that can infect all mammals, serving as intermediate hosts. The cause of congenital toxoplasmosis is transplacental transmission of the parasite to the foetus, resulting in wide range of manifestations from mild chorioretinitis to miscarriage. Its frequency can be reduced by early screening of pregnant women which is based mainly on tests for anti-Toxoplasma antibodies. We collected serum samples of 594 pregnant women (subjects) after taking their consent over a period of two years (2016-2018) and analyzed them for anti-Toxoplasma IgG by ELISA. The positive samples were then analyzed for IgG avidity test which could differentiate between recent and past infections. The seroprevalence was also correlated with the age of the subjects and their contact with cats. 162 subjects were found positive out of which only three showed a recent infection. After following up until delivery, one of them delivered a baby who had jaundice and was diagnosed with anti-Toxoplasma IgM at birth. The foetus of the second subject died in-utero, while the third woman delivered a normal baby after being given spiramycin when diagnosed with toxoplasmosis in the first trimester. It was found that most of the positive subjects had frequent contact with cats. Invasion of the parasite during third trimester resulted in death in-utero and jaundice. Most common cause of pregnancy wastage during our study was spontaneous abortions while pregnancy loss due to congenital anomalies was rare.
\end{abstract}

\section{INTRODUCTION}

It is known that Toxoplasma gondii which is an obligate intracellular protozoan parasite is able to infect all warm-blooded animals who serve as intermediate hosts and almost one-third of world's human population (Wam et al., 2016; Iddawaela et al., 2017; Khan and Khan 2018). The disease caused by this parasite is called 'toxoplasmosis' which is associated with congenital anomalies (Hoffman et al., 2012). The definitive hosts of the parasite are felids in which oocysts are produced and defecated out. Oocysts are resistant against extreme environmental conditions and become highly infectious after getting matured in soil under favourable conditions (Puvanesuaran et al., 2012; Yolken et al., 2017). Once the oocysts are ingested by mammals, they undergo development and finally give rise to tachyzoites which get propagated through the blood and consequently reach all tissues mainly the muscles, eyes, CNS and placenta thereby infecting them (Many and Koren 2006; Khan and Khan 2018). Humans can get the infection of T. gondii through two most common routes: either through ingestion of oocysts in water or food contaminated with infected cat's feces or through the ingestion of undercooked or raw meat infected with tissue cysts. If the infected individual is a pregnant woman, this infection can be transmitted through the placenta to the unborn developing foetus 
thereby obstructing its growth. When $T$. gondii crosses the placenta and reaches the foetus, it may cause congenital toxoplasmosis in the foetus. Women who acquire toxoplasmosis during pregnancy can transmit infection to their foetus through the placenta as the tachyzoites are capable of bypassing the placental blood barrier and attack the foetal organs. Tachyzoites proliferate and interfere with the developmental process in the foetus which results in foetal loss, repeated abortions and stillbirths in pregnant women. Thus, it becomes necessary to assess the immunological status of pregnant women against toxoplasmosis as consequences of congenital infection are devastating (Malarvizhi et al., 2012; Sarkar et al., 2012; Malik et al., 2014; Sarkari et al., 2014). Congenital toxoplasmosis may be present without signs or symptoms, or present with clinical symptoms like intrauterine growth restriction, hepatosplenomegaly, jaundice (Mohanty et al., 2012) or neurological and ocular manifestations like hydrocephalus, microcephaly, seizures, epilepsy, intracranial calcification, mental retardation, chorioretinitis and blindness (Rodrigues $e t a l ., 2014$ ). Other modes of infection include organ transplantation (Lopes et al., 2019) and blood transfusion (Amoo et al., 2019).

In the United States, where toxoplasmosis is considered as the second most important cause of foodborne deaths, $22.5 \%$ of the 12 years and older population is infected with Toxoplasma gondii (Hökelek et al., 2017). This parasite is widespread throughout the world with incidence as high as $95 \%$ in some populations. Areas that are situated at low altitudes and have hot and humid climates witness highest infection rates (CDC 2015). The precise seroprevalence of Toxoplasma gondii infection in India is unknown. Even so, with the advent of various diagnostic tests, it is documented to be as low as 5\% and as high as $80 \%$ in adults (Singh 2003). As per the recent reports of World Health Organisation, the incidence of congenital infection depends on the infection pressure of that particular area i.e., when the infection pressure is high, maximum women are exposed to the infection and become seropositive and when they reach the child-bearing age, they become less susceptible to the infection as they have already been exposed. When the infection pressure is low, the opposite is the case. Hence, we can say that although seronegative women are at a greater risk, the actual population that is at risk is low overall (WHO 2013; Khan and Khan 2018).

Our present study aims at finding out the seroprevalence of low avidity antiToxoplasma IgG in the blood samples of pregnant women attending the antenatal clinic of Jawaharlal Nehru Medical College, Aligarh and correlating its relationship with their age and contact with cats and to understand the antagonistic role of progesterone towards chronicity and tissue damage during pregnancy.

\section{MATERIALS AND METHODS}

The protocol was ethically approved by the Institutional Ethics Committee, Faculty of Medicine, Jawaharlal Nehru Medical College. All experiments were carried out in compliance with the relevant laws and guidelines, in accordance with the ethical standards of the Declaration of Helsinki.

Five hundred and ninety four blood samples of pregnant women were collected in sterile vials over a period of two years i.e., 2016 to 2018. An informed consent was obtained from each subject after explaining them the purpose of the study. Data regarding their medical history and socio-economic background were also obtained. The subjects were placed under different age groups viz., 21-25 years, 26-30 years, and 31-35 years. The blood samples were centrifuged at $2000 \mathrm{rpm}$ for 5 minutes to extract the serum which was stored in numbered aliquots at $-20^{\circ} \mathrm{C}$ till assayed.

ELISA was performed to find out the seroprevalence of anti-Toxoplasma IgG using human anti-Toxoplasma gondii IgG ELISA kit (ab108776, Abcam, Massachusetts U.S.A.). The assay was performed according to the manufacturer's instructions. A standard curve was plotted and the concentration of the immunoglobulin of unknown samples was determined by extrapolation. 
All serum samples found reactive were subjected to the avidity assay using Toxoplasma gondii IgG avidity test kit (TOXGA460 NovaTec, Novalisa, Ukraine) and the assay was performed according to the manufacturer's instructions.

\section{Calculations}

Equation for straight line i.e., $\mathrm{y}=\mathrm{mx}+\mathrm{c}$ which was obtained after plotting the standard curve gave the IgG concentration of unknown samples. Serum samples with concentration values exceeding $35 \mathrm{IU} / \mathrm{mL}$ were considered reactive.

The avidity results were calculated by the given formula:

[Absorbance (sample or control) Avidity reagent]

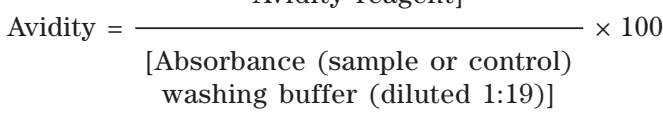

The results were interpreted as:

Avidity $\%>40 \rightarrow$ High avidity $\rightarrow$ Past infection.

Avidity $\% \leq 40 \rightarrow$ Low avidity $\rightarrow$ Acute or recent infection.

Those subjects who were found positive for low avidity anti-Toxoplasma antibodies were followed up until delivery and information about their contact with cats was also obtained.

\section{RESULTS}

All the subjects were placed under three age groups i.e. 21-25, 26-30 and 31-35 years. The overall age distribution of the subjects has been depicted in Figure 1. One hundred and sixty two serum samples out of 594 were found reactive for anti-Toxoplasma gondii IgG indicating $27.27 \%$ positivity (Figure 2 ).

The correlation between the subjects' age and the seroprevalence of $T$. gondii infection is summarized in Figure 3. The seroprevalence of the immunoglobulin was also correlated with the contact of positive women with cats and it was found that the maximum number of subjects who were positive for IgG were among those who had frequent contact with stray or a pet cat at home and the data was compared with that of seronegative pregnant women (Figure 4).

\section{STATISTICS}

The data regarding the seroprevalence among different age groups were analyzed by chi-square test where the $\chi 2$ value was found to be 15.99 while the $P$-value was 0.0003 (<0.05). Therefore, we conclude that the difference among the three age groups has a very high statistical significance. Moreover, the data regarding the contact of seropositive subjects with cats also turned out to be significantly different among the three categories $[\chi 2=204.89, P$ value $<0.00001]$.

The above data gives us an idea that out of the 162 subjects found positive for antiToxoplasma gondii IgG, only 3 were found to have acquired a recent infection. It was found that out of these 3 , one woman whose blood sample was collected in the third trimester delivered a baby who had jaundice at birth and was diagnosed positive for antiToxoplasma IgM even after treatment with

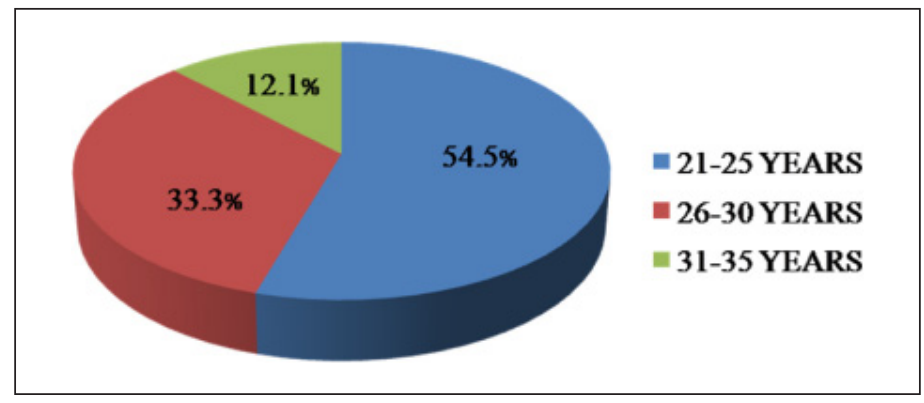

Figure 1. Proportions of the subjects in different age groups. 


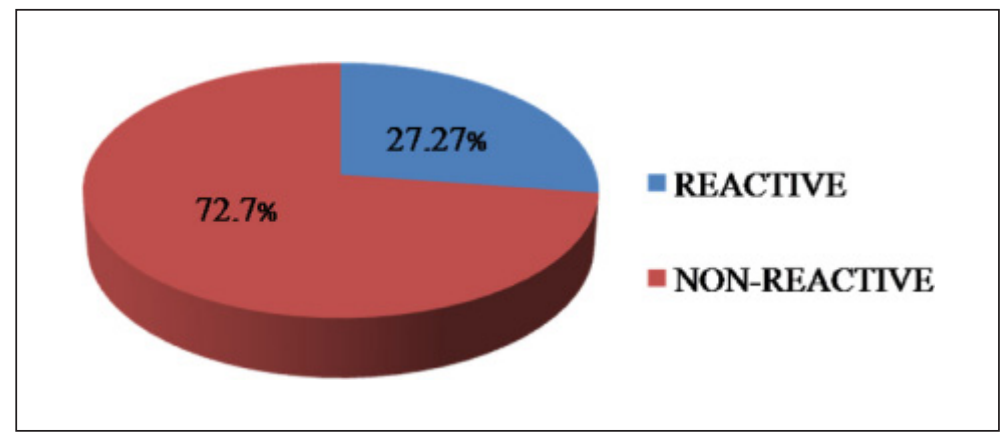

Figure 2. Seroprevalence of anti-Toxoplasma IgG in pregnant women.

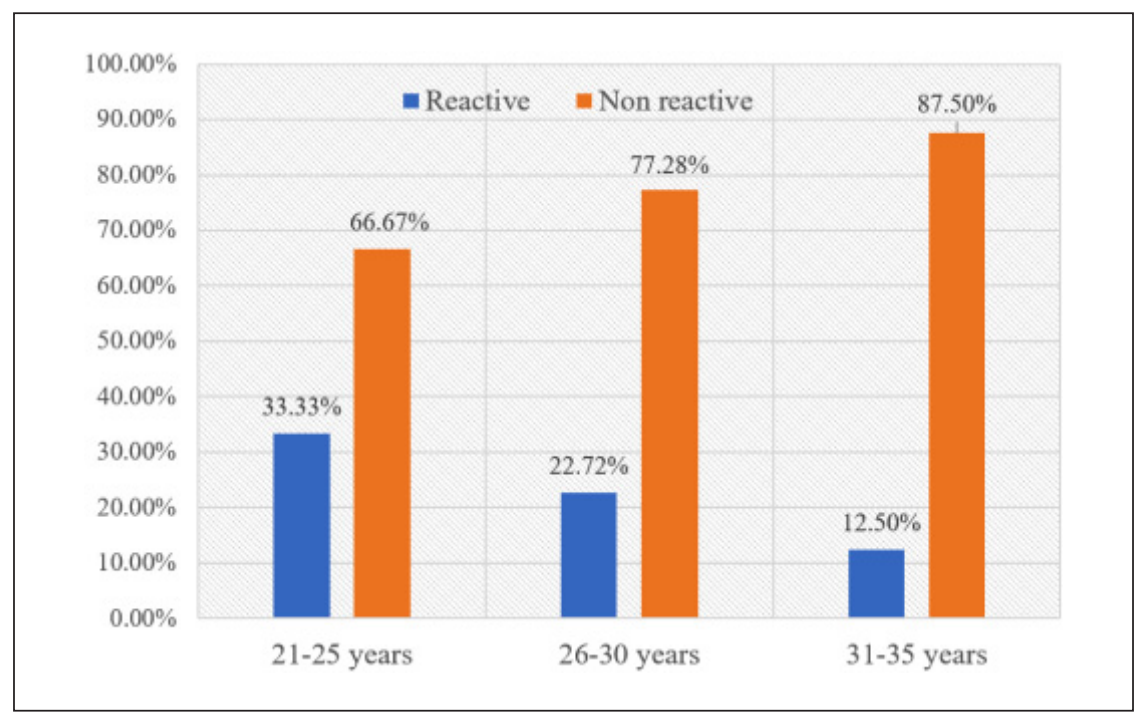

Figure 3. Seroprevalence rates among women of different age groups.

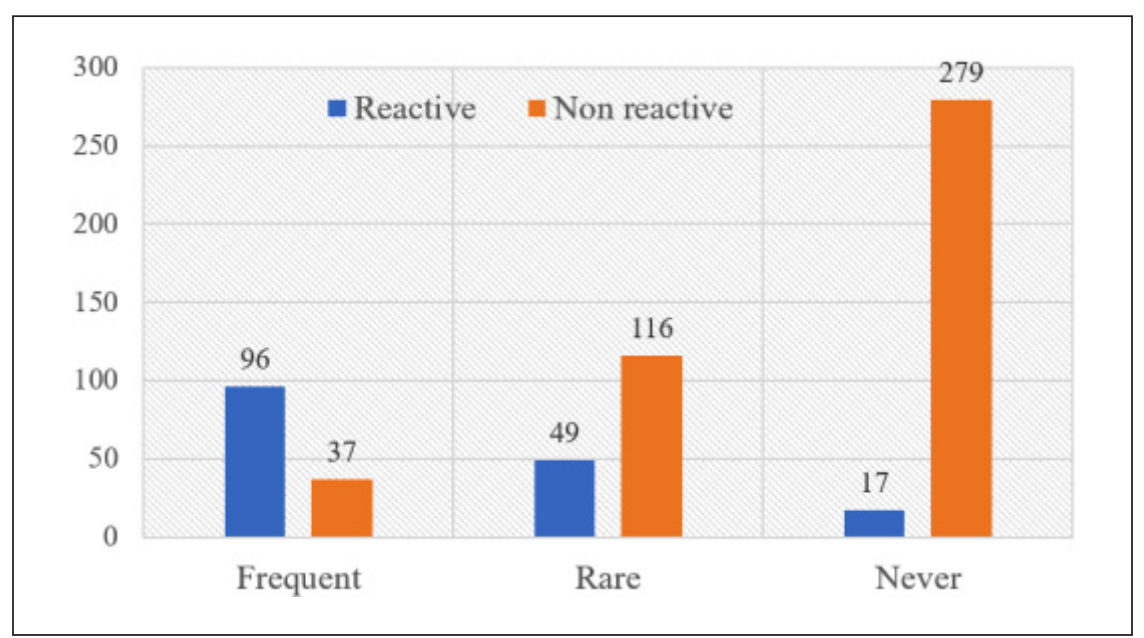

Figure 4. Seropositivity and seronegativity in pregnant women against cat contact. 
Table 1. Avidity of anti-Toxoplasma IgG among the seropositive subjects and their pregnancy outcomes

\begin{tabular}{lclcll}
\hline $\begin{array}{c}\text { Sl. } \\
\text { No. }\end{array}$ & Age & Contact with cats & $\begin{array}{c}\text { IgG } \\
\text { Concentration } \\
\text { (IU/ml) }\end{array}$ & $\begin{array}{c}\text { Avidity } \\
(\%)\end{array}$ & Fetal outcome \\
\hline 1. & 35 & $\begin{array}{l}\text { Frequent contact with } \\
\text { stray cats }\end{array}$ & 154.271 & 38.50 & $\begin{array}{l}\text { The new born baby was } \\
\text { diagnosed positive for anti- } \\
\text { Toxoplasma IgM and jaundice. }\end{array}$ \\
2. & 24 & $\begin{array}{l}\text { Frequent contact with } \\
\text { stray cats }\end{array}$ & 146.054 & 29.36 & $\begin{array}{l}\text { Intra-uterine death of the } \\
\text { foetus. }\end{array}$ \\
3. & 25 & $\begin{array}{l}\text { Contact with a pet cat } \\
\text { Conterm }\end{array}$ & 156.762 & 34.67 & $\begin{array}{l}\text { Full term normal delivery } \\
\text { (FTND) }\end{array}$ \\
\hline
\end{tabular}

pyrimethamine and sulfadiazine. In case of the second woman who was also diagnosed in the third trimester, the foetus died in-utero while the third woman delivered a normal baby whose diagnosis was done in the first trimester and treatment of spiramycin was given after diagnosis. The result of the avidity test and the pregnancy outcome is summarized in Table 1.

\section{DISCUSSION}

Toxoplasmosis is a global infection which varies widely based on social and cultural habits, climate, geographic factors and most importantly, the route of transmission. Studies have suggested that toxoplasmosis is more prevalent in warm and humid areas (Studenièová et al., 2006; Wam et al., 2016). The seroprevalence of IgM and IgG found in the first nationwide survey in humans in India was $2 \%$ and $24.3 \%$, respectively. This also varied with the geographical location with the highest prevalence rate in Southern India and the lowest in the Northern India (Dhumne et al., 2007). Since highest humidity in Aligarh prevails during the months of July to September, it favours the survival and proliferation of T. gondii oocysts (Singh et al., 2014).

Our result showed that $27.27 \%$ of the subjects with recurrent pregnancy wastage were reactive for anti-Toxoplasma IgG antibodies. Out of these, $66.66 \%$ were between 21-25 years of age, while $27.77 \%$ belonged to $26-30$ years age group and $5.55 \%$ were under 31-35 years of age. This result was in consensus with that obtained previously from India (Siddiqui et al., 2014a) and Brazil (Spalding et al., 2005) where the seroprevalence rates for the age group 21-30 years were $73.9 \%$ and $74.5 \%$, respectively. However, a few studies have reported that the prevalence of $T$. gondii infection increases with age and the reason was thought to be increasing risk of exposure (Morris and Croxson 2004; Hung et al., 2007). In Windhoek, the seroprevalence of anti- $T$. gondii IgG among urban pregnant women who attended public antenatal care in 2016 was very low (2.61\%) (Van der Colf et al., 2020) while in Almadinah Almunawwarah, it was found to be $21.3 \%$ (Imam et al., 2016). The seropositivity of toxoplasmosis in pregnant women with bad obstetric history was studied in a tertiary care hospital in Andhra Pradesh, India by ELISA and found that there is a gradual increase in the seroprevalence with advancing age (Sarkar et al., 2012). However, we have contradictory results showing that most of the pregnant women who were found positive for antiToxoplasma gondii IgG were aged between 21 and 25 years followed by women under the age group 26-30 years, while lowest seroprevalence was found in women under the age group 31-35 years. This result can be validated by the fact that most of the married women below 25 years of age came from rural background with poor hygiene facilities and had frequent contact with stray cats. Moreover, after comparing the contact of cats with seropositive and seronegative women, we found that most of them who were positive for anti-Toxoplasma 
IgG had frequent contacts with cats (stray or pet), while most of those found negative for the same did not have contact with cats at all, gives us a convincing evidence that there is a direct established relationship between toxoplasmosis and the contact of the subjects with cats. In addition, the statistical analysis of our results showed that toxoplasmosis is dependent on the age of the subject as well as on the contact of the intermediate host (in this case, pregnant woman) with the definitive host i.e., cat. Earlier studies conducted in Aligarh (Siddiqui et al., 2014b) and Tamil Nadu (Malarvizhi et al., 2012) also showed similar results. Borkakoty et al. (2007) reported higher prevalence rate in people who had low socio-economic status in Assam which was seconded by Mohammed (2011) who reported that prevalence of $T$. gondi $i$ infection decreases with the adoption of better hygiene measures. The seroprevalence of $T$. gondii infection in Indian women of child-bearing age and its association with the social and environmental factors in the 4 different regions of the country was studied by Singh et al. (2014) who concluded that the prevalence was the highest in South India (37.3\%) and the lowest in Western India (8.8\%), while the overall prevalence rate was $22.4 \%$. As there is a significant variation in the environmental and climatic conditions of these two regions, it can be said that $T$. gondii infection varies more with the environmental factors than with the age of the subject. Studies conducted in the central province of Northeast India (Borkakoty et al., 2016) and Sri Lanka (Iddawela et al., 2017) suggest that the prevalence of IgG is higher in hilly regions. In a study aimed to gather the presently available intercontinental data on $T$. gondii prevalence to analyze global trends, it was observed that the global prevalence of $T$. gondii infection varies significantly with no certain inter- and intra-continental trends which may be because of a complex interaction between evolving environmental and human factors (Molan et al., 2019). Van der Colf et al. (2020) suggested that risk factors and seroprevalence of $T$. gondii lack a significant association. Their study also concluded that there is no association between consumption of unwashed vegetables or fruits and seropositivity of $T$. gondii infection since $3.45 \%$ of the subjects who denied of consuming unwashed vegetables and fruits were found seropositive. Molan et al. (2020) also did not identify any risk factor associated with the seroprevalence of T. gondii infection.

It has been reported that immune status that develop in response to acute $T$. gondii infection and that during pregnancy are antagonistic to each other (Borges et al., 2018). The pathogen-associated molecular markers present in T. gondii such as profilin, GIPLs (Hunter and Sibley 2012) and HSP70 (Ibrahim et al., 2014) interact with Toll-like receptors (TLRs-2, 4 and 11) present on the host cells. It also has cyclophilin 18 which can bind to CCR5 resulting in the activation of neutrophils, dendritic cells and macrophages and this leads to the production of IL-12 which in turn stimulates natural killer (NK) cells to produce IFN- $\gamma$ (Borges et al., 2018). The combined action of IFN- $\gamma$ and IL-12 results in the differentiation of Th1 cells which again secrete IFN- $\gamma$ and favor the activation of M1 macrophage. These macrophages produce inducible nitric oxide synthase (iNOS) which in turn produces nitric oxide (NO) that is known to inhibit essential enzymes of the mitochondria and nucleus of the parasite that results in killing of tachyzoite stage of the parasite (Pittman and Knoll 2015). Nonetheless, excessive Th1 response results in tissue damage which is the reason behind pathology (Borges et al., 2018). It has been studied that Th2 cells and Treg cells are mainly associated with reducing inflammation during toxoplasmosis. Progesterone, which is an important maternal hormone is secreted by the breasts, endometrium, ovaries and feto-placental unit. It regulates certain immune cells so as to maintain pregnancy (Borges et al., 2018). It induces stimulation of progesterone-receptorpositive lymphocytes which in turn produce progesterone-induced blocking factor (PIBF). It is the PIBF that downregulates activity of NK cells and favours the expansion of Th2 cells. Treg cells play a key role in immune regulation, development of the 
decidua (Lee et al., 2015), acceptance of the foetus and maternal immune tolerance (Svensson-Arvelund et al., 2015; Ghaebi et $a l ., 2017)$. IFN- $\gamma$ induces the expression of the placental enzyme indoleamine-2,3dioxygenase (IDO). IDO converts an essential amino acid of pregnancy, L-tryptophan to kynurenine which favors the expansion of Treg cells (Murthi et al., 2017). Moreover, the expression of IDO is increased in the placenta during acute toxoplasmosis at late gestation (Pfaff et al., 2008). Antiinflammatory interleukins like IL-4 and IL-13 which are produced by Th2 induce the activation of M2 macrophages. Progesterone level is known to increase with increasing gestational age; however, it is downregulated during $T$. gondii infection.Therefore, the interaction between pregnancy and the immune response developing against $T$. gondii infection depends on the gestational age at which the infection occurs (Borges et al., 2018). This explains the reason as to why chances of foetal transmission increase with increasing gestational age. This is also a reason behind the fact that the severity of congenital infection reduces if the mother is infected in the later phase of gestation. The interaction between different immune cells in acute toxoplasmosis during pregnancy has been summarized in Figure 5.

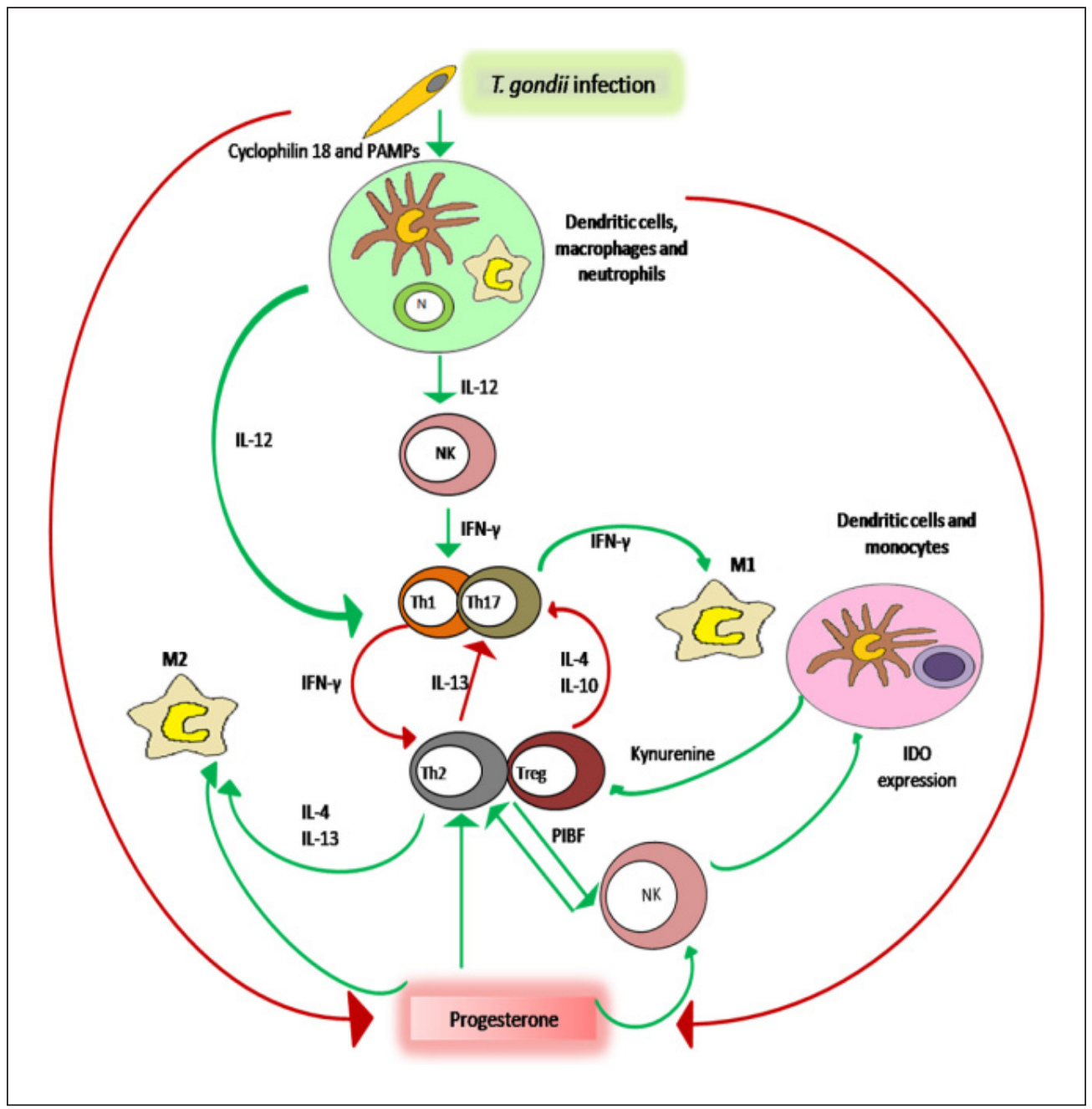

Figure 5. Interaction of the immune cells in response to acute toxoplasmosis during pregnancy. Green arrows indicate upregulation while red arrows indicate downregulation. 
The primary method of determining toxoplasmosis is the detection of Toxoplasmaspecific antibodies, the most common of which are enzyme immunoassays and immunofluorescence assay for IgG and IgM. However, determining the time of infection during pregnancy is of utmost importance in order to prevent foetal infection. Tests for the detection of immunoglobulins (IgM and IgG) produced against T. gondii are most commonly used to determine whether a pregnant woman has acquired acute infection (Khan and Khan 2018). If there is a low IgG titre and a negative IgM test in the first 24 weeks of gestation, then the infection is essentially acquired prior to gestation. Usually, detection of anti-Toxoplasmaspecific IgM antibodies is a precise indicator of a recent infection nevertheless; falsepositive IgM antibody test results have also been reported in earlier studies that is why anti-Toxoplasma IgG avidity test is more reliable for the diagnosis of a recent infection (Gras et al., 2004; Iqbal and Khalid 2007). It helps to differentiate between recent and past infections as the net antigen binding force of the antibody strengthens gradually by antigen driven B-cell selection. The antibody-antigen complex is dissociated by protein denaturing agents, including urea. The ratios of antibody titration curves of urea treated and untreated samples are used to determine the avidity result (Iqbal and Khalid 2007). In our study, we found that only 3 (1.85\%) out of the 162 seropositive subjects had low avidity IgG while the remaining 159 (98.15\%) had high avidity antibodies indicating towards past infection. We also found that mother-to-childtransmission (MTCT) of T. gondii took place in women who were diagnosed in the third trimester. However, the third subject in our study was diagnosed in the first trimester and was immediately advised to take spiramycin which is known to prevent congenital transmission of the infection. Fortunately, she delivered a normal baby who was tested negative for anti-Toxoplasma IgM and IgG. Studying the avidity of IgG in pregnant women in whom seroconversion has taken place during gestation showed that high avidity test results were associated with $T$. gondii infections acquired for at least 3-5 months.
In order to offer the pregnant women early therapy or other interventions to check congenital infection, the early diagnosis in the first trimester is of great importance (Emna et al., 2006; Reis et al., 2008; Khan and Khan 2018). Moreover, the avidity test in women during the first 16 weeks of gestation decreased the need of unnecessary treatment with spiramycin and other drugs thereby reducing the cost of the treatment as earlier observed by Siddiqui et al. (2014b). Hence, the Toxoplasma-specific antibody status of a woman before or during the first trimester of pregnancy is desirable to know. In case of toxoplasmosis, routine serological tests are highly sensitive; nevertheless, specificity varies depending on the test used. It has been documented in the earlier studies that VIDAS avidity test gives highly sensitive and specific results which make it easier to detect a recently acquired infection in IgM positive cases (Petersen et al., 2005).

Toxoplasma gondii is known to cause devastating effects if transmitted transplacentally to the foetus; therefore, its timely diagnosis during gestation is of great importance. As it is asymptomatic in immunocompetent individuals, its diagnosis in pregnant women becomes difficult, thereby increasing the chances of its transmission to the foetus. It is therefore, necessary to educate women of childbearing age about the risks associated with undercooked meat and soil contact. They also need to be made aware of the safe handling of cat litter during pregnancy. In our study, most common cause of pregnancy wastage was spontaneous abortions while pregnancy loss due to congenital anomalies was rarely obtained.

Acknowledgements. The authors would like to acknowledge the technical support extended by Dr. Ashish Yadav, Aakaar Biotechnologies Pvt. Ltd., Lucknow, India and Prof. S.M.A. Abidi, Section of Parasitology, Department of Zoology, Aligarh Muslim University, Aligarh, India. The authors would also like to thank University Grants Commission, New Delhi, India for providing financial support in the form of Maulana Azad National Fellowship to Khadija Khan. 


\section{Authors' Contributions}

$K$. Khadija conceived and carried out all the experiments and prepared the manuscript; $\mathrm{K}$. Wajihullah reviewed the manuscript; $\mathrm{K}$. Tamkeen helped in collecting blood samples; G. Naaz prepared the table and graphs. N. Ansari and A. Sana helped in the preparation of manuscript.

\section{Ethical approval}

The protocol was ethically approved by the Institutional Ethics Committee, Faculty of Medicine, Jawaharlal Nehru Medical College. All experiments were carried out in compliance with the relevant laws and guidelines, in accordance with the ethical standards of the Declaration of Helsinki. All the participants provided written informed consent to participate in the study and to have their information obtained from treating physician.

\section{Conflict of interest}

All authors declare that there is no competing interest.

\section{Funding}

This study was supported by Maulana Azad National Fellowship (Grant No. MANF-21517-ORI-71609) of the University Grants Commission, New Delhi.

\section{REFERENCES}

Amoo, A., Njaanake, K., Dada-Adegbola, H.O. \& Omosa-Manyonyi, G. (2019). Toxoplasmosis among blood donors: Unsafe blood transfusion in Ibadan, Southwest Nigeria. Journal of Applied Hematology 10: $120-125$.

Borges, M., Silva, T.M., Brito, C., Teixeira, N. \& Roberts, C.W. (2018). How does toxoplasmosis affect the maternal-foetal immune interface and pregnancy? Parasite Immunology 41(3): e12606.

Borkakoty, B., Biswas, D., Jakharia, A. \& Mahanta, J. (2016). Seroprevalence of Toxoplasma gondii among pregnant women in Northeast India. Journals of the Association of Physicians of India 64: 24-28.
Borkakoty, B.J., Borthakur, A.K. \& Gohain, M. (2007). Prevalence of Toxoplasma gondii infection amongst pregnant women in Assam India. Indian Journal of Medical Microbiology 25: 431-432.

Centres for Disease Control and Prevention, Parasites-Toxoplasmosis [Toxoplasma infection]. Accessed (2015). Available at https://www.cdc.gov/parasites/ toxoplasmosis/epi.html

Dhumne, M., Sengupta, C., Kadival, G., Rathinaswamy, A. \& Velumani, A. (2007). National seroprevalence of Toxoplasma gondii in India. Journal of Parasitology 93: 1520-1521.

Emna, S., Karim, A., Mohamed, K.C. \& Aïda, B. (2006). Difficulty in dating primary infections by Toxoplasma gondii in pregnant women in Tunisia. La Tunisie Medicale 84: 85-87.

Ghaebi, M., Nouri, M., Ghasemzadeh, A., Farzadi, L., Jadidi-Niaragh, F., Ahmadi, M. \& Yousefi, M. (2017). Immune regulatory network in successful pregnancy and reproductive failures. Biomedicine \& Pharmacotherapy 88: 61-73.

Gras, L., Gilbert, R.E., Wallon, M. \& CortinaBorja, M. (2004). Duration of the IgM response in women acquiring Toxoplasma gondii during pregnancy: implications for clinical practice and cross-sectional incidence studies. Epidemiology and Infection 132: 541548.

Hoffmann, S., Batz, M.B. \& Morris Jr. J.G. (2012). Annual cost of illness and qualityadjusted life year losses in the United States due to 14 foodborne pathogens. Journal of Food Protection 75: 12921302.

Hökelek, M. Toxoplasmosis Clinical Presentation. Accessed Sep 19, 2017. Available at https://emedicine.med scape.com/article/229969-clinical

Hung, C.C., Fan, C.K., Su, K.E., Sung, F.C., Chiou, H.Y., Gil, V., Ferreira, M., de Carvalho, J.M., Cruz, C., Lin, Y.K., Tseng, L.F., Sao, K.Y., Chang, W.C., Lan, H.S. \& Chou, S.H. (2007). Serological screening and toxoplasmosis exposure factors among pregnant women in the Demo- 
cratis Republic of Sao Tome and Principe. Transactions Royal Society of Tropical Medicine and Hygiene 101: 134-139.

Hunter, C.A. \& Sibley, L.D. (2012). Modulation of innate immunity by Toxoplasma gondii virulence effectors. Nature Reviews Microbiology 10: 766-778.

Ibrahim, H.M., Nishimura, M., Tanaka, S., Awadin, W., Furuoka, H., Xuan, X. \& Nishikawa, Y. (2014). Overproduction of Toxoplasma gondii cyclophilin-18 regulates host cell migration and enhances parasite dissemination in a CCR5-independent manner. BMC Microbiology 14: 76.

Iddawela, D., Vithana, S.M.P. \& Ratnayake, C. (2017). Seroprevalence of toxoplasmosis and risk factors of Toxoplasma gondii infection among pregnant women in Sri Lanka: a cross sectional study. $B M C$ Public Health 17: 930-935.

Imam, F.A., Azzam, A.A. \& Attia, A.A. (2016). Seroprevalence of Toxoplasma gondii among pregnant women in Almadinah Almunawwarah KSA Naglaa. The Journal of Taibah University Medical Science 11: 255-259.

Iqbal, J. \& Khalid, N. (2007). Detection of acute Toxoplasma gondii infection in early pregnancy by IgG avidity and PCR analysis. Journal of Medical Microbiology 56: 1495-1499.

Khan, K. \& Khan, W. (2018). Congenital toxoplasmosis: an overview of the neurological and ocular manifestations. Parasitology International 67: 715-721.

Lee, S.K., Kim, C.J., Kim, D.J. \& Kang, J.H. (2015). Immune cells in the female reproductive tract. Immune Network 15: 16-26.

Lopes, C.S., Silva, T.L., Cesar, J., de Almeida, N., Costa, L.V.S., Mineo, T.W.P. \& Mineo, J.R. (2019). Transmission of Toxoplasma gondii infection due to bone marrow transplantation: validation by an experimental model. Frontiers in Medicine https://doi.org/10.3389/fmed. 2019.00227

Malarvizhi, A., Viswanathan, T., Lavanya, V., Arul Sheeda Malar, S. \& Kannaiyan, M. (2012). Seroprevalence of Toxoplasma gondii in pregnant women. Journal of
Public Health and Epidemiology 4: 170177.

Malik, A., Rizvi, M., Khan, F., Khan, N., Rabbani, T. \& Khan, H.M. (2014). Toxoplasma gondii in women with bad obstetric history and infertility: a five-year study. Asian Pacific Journal of Tropical Disease 4: 236-239.

Many, A. \& Koren, G. (2006). Toxoplasmosis during pregnancy. Canadian Family Physician 52: 29-32.

Mohammed, T.K. (2011). Seroprevalence of Toxoplasma gondii among pregnant women in Baghdad city. Iraq Academy of Science Journal 24: 21-28.

Mohanty, S., Shah, I. \& Bhatnagar, S. (2012). Neonatal hepatitis with toxoplasmosis. Journal of Clinical Neonatology 1: 9697.

Molan, A., Nosaka, K., Hunter, M. \& Wang, W. (2019). Global status of Toxoplasma gondii infection: systematic review and prevalence snapshots. Tropical Biomedicine 36: 898-925.

Molan, A., Nosaka, K., Hunter, M. \& Wang, W. (2020). Seroprevalence and associated risk factors of Toxoplasma gondii infection in a representative Australian human population: The Busselton health study. Clinical Epidemiology and Global Health 8: 808-814.

Morris, A. \& Croxson, M. (2004). Serological evidence of Toxoplasma gondii infection among pregnant women in Auckland. New Zealand Medical Journal 117(1189): U770.

Murthi, P., Wallace, E.M. \& Walker, D.W. (2017). Altered placental tryptophan metabolic pathway in human fetal growth restriction. Placenta 52: 62-70.

Petersen, E., Borobio, M.V., Guy, E., Liesenfeld, O., Meroni, V., Naessens, A., Spranzi, E. \& Thulliez, P. (2005). European multicentre study of the LIAISON automated diagnostic system for determination of Toxoplasma gondii-specific immunoglobulin G (IgG) and IgM and the IgG avidity index. Journal of Clinical Microbiology 43: 1570-1574.

Pfaff, A.W., Mousli, M., Senegas, A., Marcellin, L., Takikawa, O., Klein, J.P. \& Candolfi, E. (2008). Impact of foetus and mother on 
IFN-gamma-induced indoleamine 2,3dioxygenase and inducible nitric oxide synthase expression in murine placenta following Toxoplasma gondii infection. International Journal of Parasitology 38: 249-258.

Pittman, K.J. \& Knoll, L.J. (2015). Long-term relationships: the complicated interplay between the host and the developmental stages of Toxoplasma gondii during acute and chronic infections. Microbiology and Molecular Biology 79: 387401.

Puvanesuaran, V.R., Ibrahim, N., Noordin, R. \& Balakrishnan, V. (2012). Isolation of viable Toxoplasma gondii cysts from brain samples for oral infection. European Review for Medical and Pharmacological Sciences 16: 11791183.

Reis, M.M., Tessaro, M.M. \& D'Azevedo, P.A. (2008). Toxoplasma IgM and IgG avidity in single samples from areas with a high infection rate can determine the risk of mother-to-child transmission. Revista do Instituto de Medicina Tropical de Sao Paulo 48: 93-98.

Rodrigues, I.M.X., Costa, T.L., Avelar, J.B., Amaral, W.N., Castro, A.M. \& Avelino, M.M. (2014). Assessment of laboratory methods used in the diagnosis of congenital toxoplasmosis after maternal treatment with spiramycin in pregnancy. BMC Infectious Disease 14: 349-357.

Sarkar, M.D., Anuradha, B., Sharma, N. \& Roy, R.N. (2012). Seropositivity of toxoplasmosis in antenatal women with bad obstetric history in a tertiary-care hospital of Andhra Pradesh, India. Journal of Health Population and Nutrition 30: 87-92.

Sarkari, B., Shafiei, R., Zare, M., Sohrabpour, S. \& Kasraian, L. (2014). Seroprevalence and molecular diagnosis of Toxoplasma gondii infection among blood donors in southern Iran. The Journal of Infection in Developing Countries 8: 543-547.
Siddiqui, N., Shujatullah, F., Khan, H.M., Rabbani, T. \& Khan, P.A. (2014a). IgG Avidity Antibodies against Toxoplasma gondii in high risk females of reproductive age group in India. Korean Journal of Parasitology 52: 487-491.

Siddiqui, N., Shujatullah, F., Khan, H.M., Rabbani, T. \& Khan, P.A. (2014b). Socioeconomic status and prevalence of toxoplasmosis in pregnant women with bad obstetric history. Journal of Immunology and Vaccine Technology $\mathbf{1}$ : 101-103.

Singh, S. (2003). Mother-to-child transmission and diagnosis of Toxoplasma gondii infection during pregnancy. Indian Journal of Medical Microbiology 21: 69-76.

Singh, S., Munawwar, A., Rao, S., Mehta, S. \& Hazarika, N.K. (2014). Serologic prevalence of Toxoplasma gondii in Indian women of child bearing age and effects of social and environmental factors. PLOS Neglected Tropical Disease 8: e2737.

Spalding, S.M., Amendoeira, M.R., Klein, C.H. \& Ribeiro, L.C. (2005). Serological screening and toxoplasmosis exposure factors among pregnant women in south of Brazil. Revista da Sociedade Brasileira de Medicina Tropical 38: 173177.

Studenièová, C., Benèaiová, G. \& Holková, R. (2006). Seroprevalence of Toxoplasma gondii antibodies in a healthy population from Slovakia. European Journal Internal Medicine 17: 470-473.

Svensson-Arvelund, J., Mehta, R.B., Lindau, R., Mirrasekhian, E., Rodriguez-Martinez, H., Berg, G., Lash, G.E., Jenmalm, M.C. \& Ernerudh, J. (2015). The human fetal placenta promotes tolerance against the semi-allogenic fetus by inducing regulatory T-cells and homeostatic M2 macrophages. Journal of Immunology 194: $1534-1544$. 
Torgerson, P.R. \& Mastroiacovo, P. (2013). The global burden of congenital toxoplasmosis: a systematic review. Bulletin of World Health Organization 91: 501508.

Van der Colf, B.E., Van Zyl, G.U., Noden, B.H., \& Ntriampeba, D. (2020). Seroprevalence of Toxoplasma gondii infection among pregnant women in Windhoek, Namibia, in 2016. Southern African Journal of Infectious Diseases 35: a25

Wam, E.C., Sama, L.F., Ali, I.M., Ebile, W.A., Aghangu, L.A. \& Tume, C.B. (2016). Seroprevalence of Toxoplasma gondii IgG and IgM antibodies and associated risk factors in women of child-bearing age in Njinikom, NW Cameroon. $B M C$ Research Notes 9: 406-413.
Wolfson, M.L., Schander, J.A., Bariani, M.V., Correa, F. \& Franchi, A.M. (2015). Progesterone modulates the LPSinduced nitric oxide production by a progesterone-receptor independent mechanism. European Journal of Pharmacology 769: 110-116.

Yolken, R., Torrey, E.F. \& Dickerson, F. (2017). Evidence of increased exposure to Toxoplasma gondii in individuals with recent onset psychosis but not with established schizophrenia. PLOS Neglected Tropical Disease 11: e0006040. 